\title{
A Study of Retinal Blood Vessel Extraction from the Retinal Images
}

\author{
Sandeep Singh ${ }^{1}$, Dr. Dinesh Kumar ${ }^{2}$ \\ M.Tech - CSE, Research Scholar, Guru Kashi University, Talwandi Sabo, Bathinda, Punjab \\ Associate Professor, Department of CSE, Guru Kashi University, Talwandi Sabo, Bathinda, Punjab
}

\begin{abstract}
Detecting abnormalities such as venous looping or beading sis critical for early treatment as they are in most cases indication of potentially sight-threatening retinopathy. In order to utilize these useful characteristics of retinal blood vessels, it is very important to obtain their locations and shapes accurately. Blood vessels appeared as networks of either deep red or orange-red filaments that originated within the optic disc and were of progressively diminishing width. The major problem of Patients suffering from the disease over a long period of time is more likely to develop eye problems known as diabetic retinopathy. The detection of blood vessels is a major problem in the automatic processing of retinal images. On the one hand, the vessels have certain properties such as diameter which may be the key indicators in the evolution of certain retinopathies. Two major problems in the segmentation of retinal blood vessels are the presence of a wide variety of vessel widths and the heterogeneous background of the retina. The problem is to identify the segmentation of green veins and red veins in retina.
\end{abstract}

Keyword: veins, retina, abnormalities, Blood vessels etc.

\section{INTRODUCTION}

Diabetic retinopathy is a complication of diabetes and is a major cause of blindness in developed countries. The patients might not notice a loss of vision until it became too severe, hence early diagnosis and timely treatment is vital to delay or prevent visual impair and even blindness. Retinal vessel segmentation can simplify screening for retinopathy by reducing the number of false positive results in micro aneurysm detection and may serve as a means of image registration from the same patient taken at different times by delineating the location of the optic disc and fovea. However, manual detection of blood vessels is not simple because the vessels in a retinal image are complex and have low contrast. Detecting abnormalities such as venous looping or beading sis critical for early treatment as they are in most cases indication of potentially sight-threatening retinopathy. In order to utilize these useful characteristics of retinal blood vessels, it is very important to obtain their locations and shapes accurately. Blood vessels appeared as networks of either deep red or orange-red filaments that originated within the optic disc and were of progressively diminishing width. Several approaches for extracting retinal image vessels have been developed which can be divided as; one consists of supervised classifier-based algorithms and the other utilizes tracking-based approaches. Supervised classifierbased algorithm usually comprise of two steps. First, a low-level algorithm produces segmentation of spatially connected regions. The application of mathematical morphology and wavelet transform was investigated for identification of retinal blood vessels. In a follow-up study, a two-dimensional Gabor wavelet was utilized to initially segment the retinal images.

\section{BLOOD VESSEL EXTRACTION USING MORPHOLOGICAL OPERATION}

In this method, the retinal image is taken as the input image. Then the input retinal image is pre-processed. In

pre-processing stage, the input image is resized and the green channel image is separated as the blood vessel appears brighter in the green channel image. Then morphological operation is performed on the green channel image. The primary morphological operations are dilation and erosion. The more complex morphological operations are opening and closing. Dilation is an operation that grows or thickens objects in a binary image. The specific manner and extent of this thickening is controlled by shape referred to a structuring element. Dilation is defined in terms of set operation. Erosion shrinks or thins objects in a binary image. The manner and extent of shrinking is controlled by a structuring element.

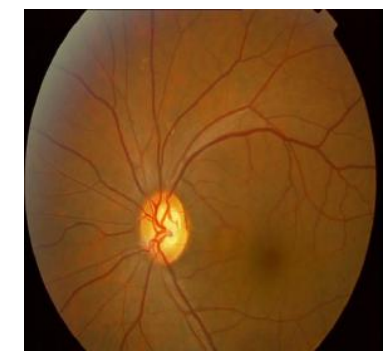

Figure 1.1 Original image

\section{LITERATURE SURVEY}

Akansha Mehrotra [2014] has proposed an automated blood vessel detection method from the fundus image. The method first performs some basic image preprocessing tasks on the green channel of the retinal image. A combination of morphological operations like top-hat and bottom-hat transformations are applied on the preprocessed image to highlight the blood vessels. Finally, the Kohonen Clustering Network is applied to cluster the input image into two clusters namely vessel and nonvessel .The performance of the proposed method is tested by applying it on retinal images from Digital Retinal 
Images for Vessel Extraction (DRIVE) database. The results obtained from the proposed method are compared with three other state of the art methods. The sensitivity, false-positive fraction (FPF) and accuracy of the proposed method is found to be higher than the other methods which imply that the proposed method is more efficient and accurate.

D. Siva Sundhara Raja [2014] have proposed The retinal image diagnosis is an important methodology for diabetic retinopathy detection and analysis. in this paper, the morphological operations and svm classifier are used to detect and segment the blood vessels from the retinal image. The proposed system consists of three stages-first is preprocessing of retinal image to separate the green channel and second stage is retinal image enhancement and third stage is blood vessel segmentation using morphological operations and svm classifier. The performance of the proposed system is analyzed using publicly available dataset.

K.Jeyasri [2013 In this paper, Retinal images play vital role in several applications such as disease diagnosis and human recognition. They also play a major role in early detection of diabetics by comparing the states of the retinal blood vessels. The detection of blood vessels from the retinal images is a tedious process. In this work a new algorithm to detect the blood vessels effectively has been proposed. Initially enhancement of the image is carried out using curvelet transform and modification of the curvelet coefficients. Since the blood vessels are distributed in various directions, morphology processing with multidirectional structuring elements are used to extract the blood vessel from the retinal images. Afterwards, morphological operator by reconstruction using multistructure elements eliminates the ridges not belonging to the vessel tree. A simple thresholding along with connected component analysis (CCA) indicates the remained ridges belonging to vessel tree. Experimental results show that the blood vessels are extracted from the retinal images with better PSNR and $96 \%$ accuracy than enhancement using other techniques.

Nidhal Khdhair El Abbadi [2013] have proposed The retinal vasculature is composed of the arteries and veins with their tributaries which are visible within the retinal image. The segmentation and measurement of the retinal vasculature is of primary interest in the diagnosis and treatment of a number of systemic and ophthalmologic conditions. The accurate segmentation of the retinal blood vessels is often an essential prerequisite step in the identification of retinal anatomy and pathology. In this study, we present an automated approach for blood vessels extraction using mathematical morphology. Two main steps are involved: enhancement operation is applied to the original retinal image in order to remove the noise and increase contrast of retinal blood vessels and morphology operations are employed to extract retinal blood vessels. This operation of segmentation is applied to binary image of top hat transformation. The result was compared with other algorithms and give better results. 


\section{CONCLUSION}

The major problem of Patients suffering from the disease over a long period of time is more likely to develop eye problems known as diabetic retinopathy. The detection of blood vessels is a major problem in the automatic processing of retinal images. On the one hand, the vessels have certain properties such as diameter which may be the key indicators in the evolution of certain retinopathies. To resolve the segmentation of retinal blood vessels are the presence of a wide variety of vessel widths and the heterogeneous background of the retina. The different researcher views are studied to review the retinal images problem and blood vessel problem. In the Future the Red and Green blood vessel is extracted with the help of KCN and morphological operators.

\section{REFERENCES}

[1]. Akansha Mehrotra "Blood Vessel Extraction For Retinal Images Using Morphological Operator and KCN Clustering” IEEE 2014.

[2]. K.Jeyasri "Detection of Retinal Blood Vessels for Disease Diagnosis" International Journal of Advanced Research in Computer Science and Software Engineering, Volume 3, Issue 3, March 2013 ISSN: 2277 128X .

[3]. D.Siva Sundhara Raja "PERFORMANCE ANALYSIS OF RETINAL IMAGE BLOOD VESSEL SEGMENTATION" Advanced Computing: An International Journal (ACIJ), Vol.5, No.2/3, May 2014.

[4]. Nidhal Khdhair El Abbadi "BLOOD VESSELS EXTRACTION USING MATHEMATICAL MORPHOLOGY" Journal of Computer Science 9 (10): 1389-1395, 2013,ISSN: 1549-3636.

[5]. X Xu, M. Niemeijer, Q. Song, M. Sonka, M. K. Garvin, J. M. Reinhardt, and M. D. Abràmoff, - Vessel boundary delineation on fundus images using graph-based approach,\| IEEE Transactions on Medical Imaging, vol. 30, no. 6, June 2011.

[6]. B. S. Y. Lam, Y, Gao, and A. W.-C. Liew, -General retinal vessel segmentation using regularization based multiconcavity modeling,\| IEEE Transactions on Medical Imaging, vol. 29, no. 7, July 2010.

[7]. M. A. Palomera-P'erez, M. Elena Martinez-Perez, Hector Ben'1tez$\mathrm{P}^{\prime}$ erez, and Jorge Luis Ortega-Arjona, - Parallel multiscale feature extraction and region growing: application in retinal blood vessel detection\|, IEEE Transactions on Information Technology in Biomedicine, vol. 14, no. 2,March 2010.

[8]. R. Horaud and T. Skordas, - Stereo correspondence through feature grouping and maximal cliques, IEEE Trans. Pattern Anal. Machine Intell, vol. 11, pp. 1168-1180, Nov. 1989.[5] W. M. Well, -Statistical approaches to feature-based object recognition, Int. J. Comput. Vision, vol.21, no. 1, pp. 63-98, 1997.

[9]. S. Chaudhuri, S. Chatterjee, N. Katz, M. Nelson, and M. Goldbaum, -Detection of blood vessels inretinal images using two-dimensional matched filters, ॥ IEEE Trans. Med. Imag., vol. 8, pp. 263-269,Sept. 1989

[10]. A. Can, H. Shen, J. N. Turner, H. L. Tanenbaum, and B. Roysam, -Rapid automated tracing and feature extraction from retinal fundus images using direct exploratory algorithms, IEEE Trans.Inform. Technol. Biomed., vol. 3, pp. 125-138, June 1999. 